\title{
ANTROPOMETRIA APLICADA AOS OPERADORES DE TRATORES AGRÍCOLAS
}

\author{
ANTHROPOMETRICS APPLIED TO THE AGRICULTURAL \\ TRACTORS OPERATORS
}

\author{
José Fernando Schlosser $^{1}$ Henrique Debiasi ${ }^{2}$ Geovano Parcianello ${ }^{3}$ \\ Lisandro Rambo ${ }^{3}$
}

\section{RESUMO}

$O$ objetivo deste trabalho foi determinar o padrão antropométrico dos operadores de tratores agrícolas da Depressão Central do Rio Grande do Sul. Os operadores de tratores agrícolas foram medidos por meio de um painel constituído por duas chapas metálicas, permanecendo durante as medições em pé, eretos, com o mínimo de roupa possível e em contato com o painel. As variáveis medidas foram as seguintes: altura do corpo; altura ao nível dos olhos; altura ao nível dos olhos sentado; altura do cotovelo; alcance do braço; alcance da mão; distância pé-patela; apoio do assento. Os dados obtidos demonstram que há diferenças entre o biótipo do operador utilizado pela indústria de tratores agrícolas e o do operador da região, de forma que este último apresentou, para todas as medidas, à exceção do apoio do assento, uma média maior. Observa-se também que, para cada medida, os limites inferior e superior do intervalo onde se encontram $90 \%$ dos operadores avaliados foram, respectivamente, menores e maiores que o padrão utilizado pela indústria, caracterizando uma maior variação. Levando-se em consideração as diferenças existentes entre o perfil antropométrico dos operadores de tratores agrícolas da Depressão Central do Rio Grande do Sul e os parâmetros utilizados pela indústria, pode-se confirmar a hipótese de que os tratores agrícolas que se encontram atualmente em comercialização no Brasil podem não oferecer $o$ conforto necessário ao operador desta região .

Palavras-chave: antropometria, tratores, ergonomia

\section{SUMMARY}

The objective of this work was to evaluate the anthropometric measures of the agricultural tractor operators of the Central Region of Rio Grande do Sul State. The agricultural tractor operators were measured through a panel that was made of two metallic plates. During the measurement, they were standing, wearing the minimum amount clothes possible, and touching the panel. The measures body height, eyes level height (standing), eyes level height (sitting), elbow height, arms reach, hands reach, kneecap-foot length and seat length were taken. The data showed that there are differences between the operators' measures used by industry and those taken in this research. The anthropometric measures of the analyzed operators were usually higher than the industrial parameters. For each measure, the lower and bigger upper limits, that included $90 \%$ of the operators measured, were lower and higher, respectively, to those used as industrial parameters. This characterizes a major variation. Based on the differences, the commercial agricultural tractors in Brazil can not offer the necessary comfort and ergonomics to the operator of the region.

Key words: antropometrics, tractors, ergonomics.

\section{INTRODUÇÃO}

A intensificação do uso de máquinas agrícolas começou, no Brasil, a partir da década de 60, fruto do processo de modernização da agricultura. Entre essas máquinas, destaca-se o trator agrícola, que é considerado por alguns autores como sendo a base ou eixo da mecanização agrícola moderna (SCHLOSSER, 1998).

Em relação ao trabalho manual, o uso do trator agrícola reduziu de forma significativa a carga

\footnotetext{
${ }^{1}$ Engenheiro Agrônomo, Doutor, Professor Titular do Departamento de Engenharia Rural, Centro de Ciências Rurais (CCR), Universidade Federal de Santa Maria (UFSM), Campus Universitário, Camobi, 97.105-900, Santa Maria, RS. E-mail: schlosse@ccr.ufsm.br. Autor para correspondência.

${ }^{2}$ Engenheiro Agrônomo, Professor substituto do Departamento de Engenharia Rural, CCR, UFSM. Mestrando do Programa de Pósgraduação em Engenharia Agrícola da UFSM.

${ }^{3}$ Engenheiro Agrônomo, Mestrando do Programa de Pós-graduação em Agronomia da Universidade Federal do Rio Grande do Sul.

Recebido para publicação em 30.04.01. Aprovado em 30.01.02
} 
física à qual o trabalhador encontrava-se submetido. Entretanto, os operadores de tratores agrícola continuam expostos a uma determinada carga física e, neste caso, também mental, pois a operação de um trator exige o controle simultâneo de diversas variáveis referentes ao trabalho, conforme explica MÁRQUEZ (1990). O esforço físico e mental leva à fadiga, o que diminui a capacidade de concentração do operador, aumentando, em conseqüência, a ocorrência de acidentes de trabalho, que podem resultar em erros (MÁRQUEZ, 1990). Da mesma forma, diminui a produtividade do trabalho (PURCELL, 1996; WITNEY, 1988) e colabora para uma maior incidência de doenças ocupacionais. A intensidade do esforço físico e mental depende, em grande parte, das características ergonômicas dos tratores agrícolas (PURCELL, 1996).

A adaptação do posto de operação ao operador pode ocorrer de duas maneiras distintas. A primeira delas refere-se à incorporação ao projeto de itens qualitativos de conforto, como cabinas, dispositivos eletrônicos de controle, dispositivos absorvedores de vibrações, entre outros. Geralmente, a incorporação destes itens implica um aumento considerável do custo da máquina ao agricultor. A agricultura brasileira, em crise desde meados da década de 80, não tem condições de suportar este diferencial de custo. Assim, para ter competitividade neste mercado, através da redução do valor de venda do produto, as indústrias importam os projetos originais de suas matrizes e retiram os itens relacionados ao conforto (SCHLOSSER, 1998). A outra forma de adaptação da máquina ao homem relaciona-se à correta disposição e dimensionamento de todos os componentes do posto de operação, como comandos, volante de direção, assento, estruturas de proteção e vias de acesso e saída. Contrariamente à incorporação de itens qualitativos, esta maneira de adaptação não resulta em grandes aumentos nos custo e, embora não resolva todos os problemas relacionados à qualidade ergonômica dos tratores, colabora para uma significativa melhoria nas condições de conforto oferecidas ao operador e, em conseqüência, para uma diminuição na ocorrência de acidentes e de doenças ocupacionais e para um aumento na produtividade do trabalho.

Para que o projeto do trator agrícola possa contemplar o correto posicionamento e dimensionamento dos componentes do posto de operação, a ergonomia recorre a outra ciência, a antropometria. Segundo MINETTE (1996), a antropometria é a parte da antropologia física que estuda as dimensões do corpo humano. As medidas corporais de que trata a antropometria são usadas para definir a localização dos componentes do posto de operação, de forma que indivíduos de diferentes tamanhos tenham fácil acesso e saída ao posto de operação, bem como consigam alcançar e acionar, com o mínimo esforço e de forma a manter uma postura corporal correta, todos os comandos (volante, pedais dos freios e da embreagem, acelerador, entre outros). ROBIN (1987) expõe ainda que a colocação de todas as partes constituintes do posto de operação deve ser tal que haja espaço livre ao redor do operador para que este possa se movimentar sem dificuldade.

As dimensões que caracterizam o posto de operação dos tratores agrícolas encontram-se normatizadas em nível internacional. Entre essas normas, destacam-se a norma ISO 3462 - 1979 (Tratores e Máquinas Agrícolas - Ponto de Referência do Assento - Método de Determinação), a ISO 4253 - 1977 (Tratores Agrícolas - Banco do operador - dimensões) e a UNE 68 - 046 - 83 (Tratores Agrícolas - Acessos, Saídas e Posto do Condutor - Medidas).

Os padrões definidos por estas normas estão de acordo com as medidas antropométricas dos operadores europeus e norte-americanos que, a princípio, podem diferir das medidas dos operadores de outros países, como o Brasil (SIQUEIRA, 1976). As variações no padrão antropométrico podem ocorrer inclusive dentro de um mesmo país, conforme a região considerada. Assim, um trator agrícola, cujo posto de operação esteja dimensionado conforme os padrões definidos pelas normas internacionais, pode proporcionar um ambiente de trabalho inadequado ao operador brasileiro.

Poucos são os estudos antropométricos de operadores de tratores agrícolas executados em países em desenvolvimento. Entre eles, pode-se destacar um levantamento feito na Índia (YADAV et al., 1999), onde foram determinadas 24 medidas corporais de operadores de tratores agrícolas, somente do sexo masculino. Estes autores observaram que os operadores analisados foram menores que os norte americanos e europeus em quase todas as dimensões avaliadas.

No Brasil, não tem sido relatada nenhuma pesquisa que vise determinar o padrão antropométrico de operadores de tratores. Alguns estudos antropométricos foram efetuados (IIDA \& WIERZBICKI, 1973; FERREIRA, 1988; MINETTE, 1996), mas para populações que não eram constituídas de operadores de tratores agrícolas, bem como englobando indivíduos de outras regiões que não a Depressão Central do Rio Grande do Sul. Comparando-se as medidas obtidas nas referidas pesquisas, com as dos países 
desenvolvidos (Kroemer apud GRANDJEAN, 1988), observa-se que os brasileiros das regiões abrangidas pelas pesquisas são maiores para algumas medidas e menores para outras. Porém, para a região da Depressão Central do Estado do Rio Grande do Sul, considerando-se operadores de tratores agrícolas, este comportamento pode não ser o mesmo pois, conforme já foi exposto anteriormente, as medidas antropométricas podem variar de região para região.

Assim, o objetivo deste trabalho foi determinar o padrão antropométrico dos operadores de tratores agrícolas da Depressão Central do Rio Grande do Sul, e compará-lo com o dos países desenvolvidos.

\section{MATERIAL E MÉTODOS}

Foram determinadas oito medidas corporais de operadores de tratores agrícolas da região da Depressão Central do Estado do Rio Grande do Sul. Essas medidas podem ser melhor visualizadas na figura 1 .

Para a obtenção destas medidas, foi utilizado um painel constituído por duas chapas metálicas lisas de $2 \mathrm{~m}$ de altura, tendo uma delas $1 \mathrm{~m}$ e a outra $2 \mathrm{~m}$ de largura, posicionadas de tal maneira a formar um ângulo de $90^{\circ}$ entre si e com o piso. As referidas chapas foram quadriculadas com precisão de $0,5 \mathrm{~cm}$, através de pintura, para possibilitar a tomada das medidas antropométricas de cada indivíduo.

A análise estatística constou do cálculo dos percentis, nos níveis 5, 50 e 95\%. Também foram determinados o desvio padrão, o coeficiente de variação, a média e o intervalo da medida no qual se encontram $90 \%$ dos indivíduos, sendo que estas duas últimas determinações foram utilizadas para efeito de comparação com dados antropométricos referentes aos países desenvolvidos. Essa comparação foi feita através do teste $\mathrm{t}$, a um nível de $5 \%$ de probabilidade de erro.

\section{RESULTADOS E DISCUSSÃO}

Os dados antropométricos dos operadores de tratores agrícolas da região da depressão central da região sul são apresentados nas tabelas 1 e 2 .

Os resultados demonstraram, em primeiro lugar, que há diferenças entre o biótipo geral dos operadores dos países desenvolvidos e o dos operadores da região abrangida por esta pesquisa (Tabela 1), confirmando os dados obtidos por IIDA \& WIERZBICKI (1973), FERREIRA (1988) e MINETTE (1996). Este último foi significativamente maior (teste $\mathrm{t}$ em $5 \%$ de probabilidade de erro) para a altura do nível dos olhos em pé e sentado e para o alcance do braço e da mão. As maiores diferenças ocorreram para o alcance do braço (média de $89,6 \mathrm{~cm}$ para os operadores brasileiros, contra $82,0 \mathrm{~cm}$ dos operadores dos países desenvolvidos) e também, para o alcance da mão $(53,5 \mathrm{~cm}$ e $47,0 \mathrm{~cm}$, respectivamente, para os operadores brasileiros e dos países desenvolvidos). Com relação à estatura, à altura do cotovelo e à distância pé-patela, não houve diferenças significativas entre as populações analisadas. A única medida para a qual os operadores dos países desenvolvidos obtiveram valores significativamente maiores foi para o apoio do assento (50,0 cm contra $45,8 \mathrm{~cm}$ dos operadores da região).
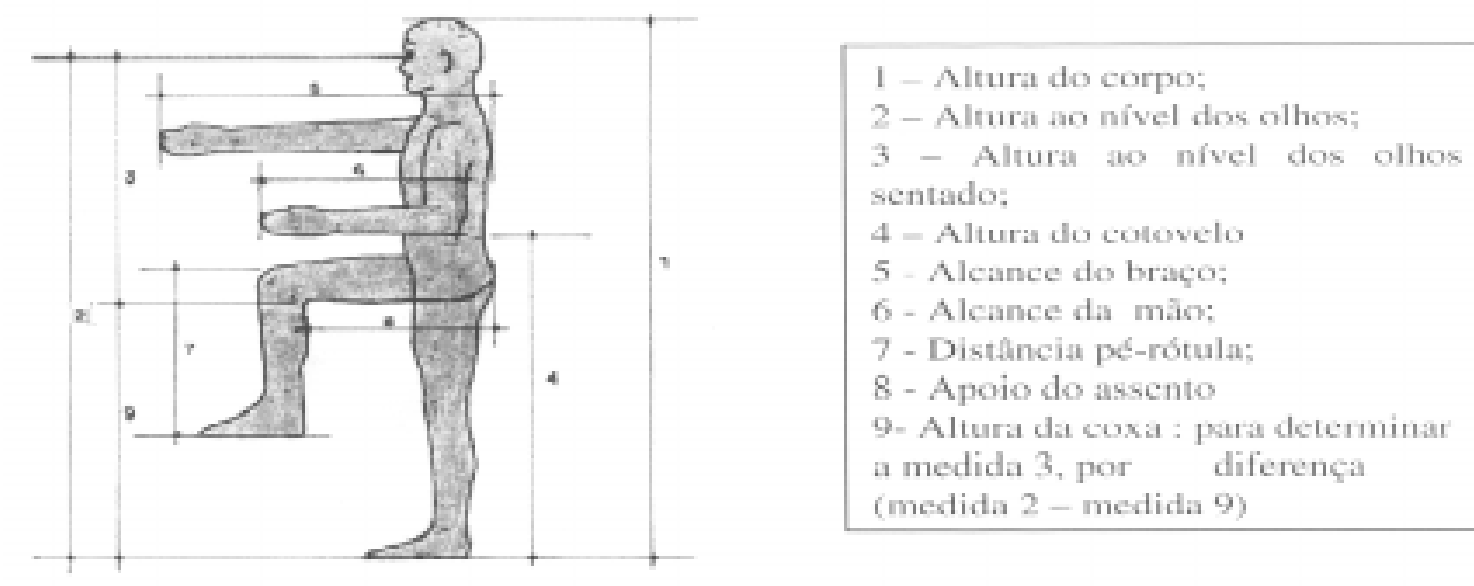

Figura 1 - Esquema representativo das medidas antropométricas a serem determinadas. 
Tabela 1 - Comparação do padrão antropométrico entre os operadores de tratores agrícolas de um país desenvolvido e a região da Depressão Central do Rio Grande Sul.

\begin{tabular}{lcccc}
\hline \multirow{2}{*}{\multicolumn{1}{c}{ Medida }} & \multicolumn{2}{c}{ País Desenvolvido } & \multicolumn{2}{c}{ Depressão Central } \\
\cline { 2 - 5 } & Média & Intervalo & Média & Intervalo \\
\cline { 2 - 5 } & \multicolumn{3}{c}{$90 \%$} & $90 \%$ \\
\hline Altura do corpo (cm) & 172 & $160-184$ & 173,9 & $157,9-186,5$ \\
Altura ao nível dos olhos & 161 & $150-172$ & $164,6^{*}$ & $150,6-174,4$ \\
em pé (cm) & & & & \\
Altura ao nível dos olhos & 79 & $73-85$ & $82,0^{*}$ & $70,0-92,6$ \\
sentado (cm) & & & & \\
Altura do cotovelo (cm) & 106 & $98-114$ & 107,9 & $98-116,5$ \\
Alcance do braço (cm) & 82 & $75-87$ & $89,6^{*}$ & $82,6-96,4$ \\
Alcance da mão (cm) & 47 & $43-51$ & $53,5^{*}$ & $47,7-66,9$ \\
Distância pé-patela (cm) & 55 & $51-59$ & 55,2 & $45,0-64,3$ \\
Apoio do assento (cm) & 50 & $46-54$ & $45,8^{*}$ & $39,0-52,2$ \\
Massa corporal (kg) & - & - & 74,9 & $58,0-89,0$ \\
\hline
\end{tabular}

*Diferenças entre médias significativas pelo teste $\mathrm{t}, \alpha=5 \%$

alcance do braço e da mão que os operadores de países desenvolvidos. Entretanto, estas diferenças não se constituem em problemas pois se os comandos estiverem na área de alcance normal e/ou máximo para os operadores oriundos de países desenvolvidos, o estarão também para os operadores analisados neste levantamento.

O posicionamento vertical dos comandos é definido também em função do comprimento do braço. Eles devem estar localizados de forma que o operador consiga alcançá-los sem sair de sua posição normal. A distância vertical máxima do nível do ombro do operador até o comando deve ser igual ao comprimento do braço. Como para essa dimensão o operador da região analisado foi maior, se os comandos estiverem bem posicionados no sentido vertical em relação aos operadores de países europeus, o estarão também para os operadores desta região.

A altura ao nível dos olhos sentado interfere diretamente no campo visual do operador. As diferenças encontradas no perfil antropométrico com relação a esta variável, embora significativas, não implicam mudanças no projeto do posto de operação. Isto pode ser justificado pelo fato de que, para um mesmo trator, quanto maior a altura do nível dos olhos do operador em relação à plataforma de operação, melhor é a visibilidade. Assim, o operador analisado nesta pesquisa, para um mesmo trator, terá um campo visual mais amplo que os dos países desenvolvidos.

$\mathrm{O}$ apoio do assento, por sua vez, é uma medida que

O alcance da mão e do braço interfere diretamente no posicionamento dos controles operados pelas mãos. Para que os controles de acionamento freqüiente (hidráulico, de controle remoto, etc.) possam ser considerados bem localizados no sentido horizontal, eles devem estar posicionados dentro da área de alcance normal, que é delimitada pelo semicírculo de raio igual ao alcance da mão. Já os controles acionados de maneira esporádica devem estar dentro da área de alcance máximo. Esta é delimitada pelo semicírculo de raio igual ao alcance do braço. Conforme já foi exposto anteriormente, os operadores medidos nesta pesquisa foram significativamente maiores para $o$ influencia na definição do comprimento do assento do operador. O comprimento do assento deve ser tal que possibilite o acionamento dos pedais da embreagem, freios e acelerador de maneira rápida e com o mínimo de esforço, sem que o operador tenha que sair de sua posição normal. Como os operadores desta região foram menores para esta dimensão, o comprimento do assento deve ser diminuído em relação ao que é recomendado para os operadores de países desenvolvidos. Caso essa especificidade não seja atendida, o operador terá que sair de sua posição normal de trabalho para poder acionar os controles operados pelos pés, obrigando-se assim a se movimentar com maior freqüência e a tirar o apoio 
do encosto vertical do assento. Este fato aumentará o nível de fadiga ao qual o operador encontra-se submetido durante a jornada de trabalho, conforme expõem MÁRQUEZ (1990) e PURCELL (1996).

A distância pé-patela é usada para definir a altura do assento em relação à plataforma de operação. Essa altura deve ser tal que o operador mantenha pés sempre apoiados e tenha fácil acesso aos controles operados pelos pés. Como essa medida não diferiu entre as das populações analisadas, pode-se inferir que o posicionamento vertical e horizontal do assento em relação aos comandos operados pelos pés, definido pelas normas internacionais, está de acordo com o padrão do operador brasileiro desta região.

Outro fator importante a ser avaliado é a amplitude dos valores referentes às diferentes dimensões. Para todas as medidas analisadas, os operadores brasileiros da região da Depressão Central do Rio Grande do Sul apresentaram uma maior variação. Isto pode ser comprovado através dos valores que delimitam o intervalo que abrange $90 \%$ dos operadores medidos (Tabela 1). Para a grande maioria das medidas, os limites superiores e inferiores do intervalo foram, respectivamente, maiores e menores que o intervalo onde se encontram 90\% dos operadores pertencentes aos países desenvolvidos. Dessa forma, os componentes do posto de operação passíveis de regulagem quanto ao posicionamento vertical e horizontal, como o volante de direção e o banco, devem permitir um maior intervalo de regulagem.

É importante salientar que os limites inferior e superior que definem o intervalo que abrange $90 \%$ dos operadores são, respectivamente, o valor de $5 \%$ e $95 \%$ percentis (Tabela 2). O percentil significa a proporção da população cuja medida é inferior a um determinado valor. Esses limites não são muito adequados para a definição do intervalo de regulagem, pois não há correlação bem definida entre as diferentes dimensões dos operadores, conforme YADAV et al. (1999). Neste sentido, um determinado operador pode estar dentro do nível de $5 \%$ percentis para o alcance do braço, mas dentro do nível de $95 \%$ para a distância pépatela.

Assim, optou-se por seguir outra metodologia (YADAV et al., 1999). Os limites inferior e superior de cada medida foram definidos, respectivamente, através do cálculo da média dos $10 \%$ menores e maiores dos operadores medidos. Os resultados são apresentados na tabela 3 .
Comparando-se os limites apresentados na figura 1 com os níveis de 5 e 95\% percentis, nota-se que há diferenças. Por exemplo, para a distância pé-patela, o intervalo de $90 \%$ definido em função dos níveis 5 e $95 \%$ percentis vai de 45,0 a $64,3 \mathrm{~cm}$. Para a mesma medida, considerando os resultados apresentados pela tabela $3, \mathrm{o}$ intervalo vai de 44,87 a $66,75 \mathrm{~cm}$.

abela 3 - Valores a serem usados como base para a definição de intervalos de regulagem, com base no padrão antropométrico dos operadores de tratores agrícolas da região da Depressão Central do Rio Grande do Sul.

\begin{tabular}{lcc}
\multicolumn{1}{c}{ Medida } & $\begin{array}{c}\text { Limite inferior } \\
(\mathrm{cm})\end{array}$ & $\begin{array}{c}\text { Limite superior } \\
(\mathrm{cm})\end{array}$ \\
\hline Altura do corpo (cm) & 160,0 & 185,5 \\
Altura ao nível dos olhos em pé $(\mathrm{cm})$ & 152,4 & 175,5 \\
Altura ao nível dos olhos sentado $(\mathrm{cm})$ & 70,6 & 92,9 \\
Altura do cotovelo $(\mathrm{cm})$ & 95,8 & 117,5 \\
Alcance do braço $(\mathrm{cm})$ & 83,0 & 96,8 \\
Alcance da mão $(\mathrm{cm})$ & 45,8 & 66,8 \\
Distância pé - patela $(\mathrm{cm})$ & 44,9 & 95,0 \\
Apoio do assento $(\mathrm{cm})$ & 39,8 & 54,3 \\
Massa corporal $(\mathrm{kg})$ & 60,5 & 88,9 \\
\hline
\end{tabular}

\section{CONCLUSÕES}

O padrão antropométrico dos operadores de tratores agrícolas da região da Depressão Central do Rio Grande do Sul é comparativamente diferente ao dos países desenvolvidos. Assim, conclui-se que há necessidade de algumas modificações no projeto dos tratores, em relação àqueles adequados aos operadores de países desenvolvidos.

\section{REFERÊNCIAS BOBLIOGRÁFICAS}

FERREIRA, D.M.P. Pesquisa antropométrica e biomecânica dos operários da indústria de transformação - RJ: I Medidas para postos de trabalho. Rio de Janeiro : Instituto Nacional de Tecnologia, 1988. 128p.

GRANDJEAN, E. Manual de ergonomia adaptando o homem ao trabalho. 4.ed. Porto Alegre : Artes Médicas, 1998. Cap.4: Medidas do corpo como parâmetros para projeto: p.39-44.

IIDA, I., WIERZBICKI, H. A. J. Ergonomia. São Bernardo do Campo : Cultura, 1973. 292p.

INTERNATIONAL ORGANIZATION FOR STANDARDIZATION. Agricultural tractors - operator's seating accommodation dimensions. ISO 4253-1977. Genève, 1977. 17p.

INTERNATIONAL ORGANIZATION FOR STANDARDIZATION. Agricultural tractors and machinery - seat reference point method of determination. ISO 3462. Genève, 1979. 21p.

INSTITUTO ESPAÑOL DE RACIONALIZACION Y NORMALIZACIÓN. Tractors agrícolas - accesos, salidas y puesto del conductor - medidas. UNE 68-046-83. Madrid, Espanha : IRANOR, 1983. 14p. 
MÁRQUEZ, L. Solo tractor'90. Madrid : Laboreo, 1990. Cap.4: Ergonomía y seguridad en los tractores: p.146-207.

MINETTE, J. L. Análise de fatores operacionais e ergonômicos na operação de corte florestal com motoserra. Viçosa, 1996, 211p. Tese (Doutorado em Ciência Florestal) - Curso de Pós graduação em Ciência Florestal, Universidade Federal de Viçosa, 1996.

ROBIN, P. Segurança e ergonomia em maquinaria agrícola tratores agrícolas. São Paulo : Instituto de Pesquisas Tecnológicas do Estado de São Paulo - IPT, 1987. p.11-15.

PURCELL, W.F.H. Human factors in tractor design. In LILJEDAHL, J.B., TURNQUIST, P.K., SMITH, D.W. Tractors and their power units. Saint Joseph : ASAE, 1996. Cap.9, p.203-239.
SCHLOSSER, J.F. Tratores agrícolas. Santa Maria : Núcleo de Ensaios de Máquinas Agrícolas, Centro de Ciências Rurais, UFSM, 1998. 64p. (Série Técnica-Módulo II).

SIQUEIRA, C.A.A. Um estudo antropométrico de trabalhadores brasileiros. Rio de Janeiro : COPPE/UFRJ, 1976. 53p.

WITNEY, B. Choosing and using farm machines. Harlow : Longman Scientific and Technical, 1988. p.28-94.

YADAV, R., TEWARI, V. K., PRASAD, N., et al. An anthropometric model of indian tractor operators. Agricultural Mechanization in Asia, Africa and Latin America, v.30, n.1, p.25-28, 1999. 